\title{
Flow in Membrane Filter Simulated as Microchannel Flow with Diaphragm
}

\author{
Liu, H. L., Xie, C., Shen, C., Fan, J. * \\ Laboratory of High Temperature Gas Dynamics \\ Institute of Mechanics, Chinese Academy of Sciences
}

\begin{abstract}
Axisymmetrical and three dimentional fows through single circular and rectangular microchannel with diaphragm are studied in this paper to imitate the performance of micro-machined membrane particle filter with grid of through holes. The microchannel flows are studied using both the DSMC method and the information preservation (IP) method which yield the result of pressure drop across the diaphragm in better agreement with the experimental data for the membrane filter, than the NS equation calculation with slip boundary condition.
\end{abstract}

\section{INTRODUCTION}

Micromachined membranes with perforations are widely used for filtering and collecting particles of the size from 1 to 10 microns. Fluid dynamic performance of such filters was investigated recently both experimentally and numerically in [1]. Fabricated by deposition and etching membranes with regularly arranged perforations were obtained with circular, rectangular and hexagonal hole shapes. The opening area factor $\beta$ (defined as the ratio of the area of the holes to the total area of the membrane) varied between $4 \%$ and $45 \%$, and the pressure drop was measured versus flow rate and opening factor. Numerical calculation was carried out by solving the N-S equation both with and without slip boundary conditions. The calculated pressure drop results of N-S equation with and without slip were much higher than the measured data. The authors appeal that more work of fluid mechanics nature is needed to resolve this discrepancy.

In the present paper single circular and rectangular microchannel with diaphragm having the same opening factor as the membrane filter are used in flow simulation to imitate the performances of membrane filter with grid of through holes. DSMC method ${ }^{[2]}$ is used to simulate the flow in the microchannel. As the microchannel imitates only one flow tube in the membrane filter flow and the flow tubes exert no viscous action on each other, the side walls are assumed to be specularly reflecting. Such a simulation yields the pressure drop result that is in agreement with the experimental data. The Information Preservation (IP) method ${ }^{[3]}$ incorporated with DSMC aimed to reduce the large noise to useful signal ratio is introduced to compare the flow field results with DSMC method. Excellent agreement with DSMC calculation and experimental data is obtained. In the following the DSMC simulation and the IP procedure are described together with the results obtained and discussions.

\section{DSMC SIMULATION}

DSMC is a method proceeding from the physical simulation of the motion of large number of representative particles. The particles' positions and velocities are modified owing to their motions and collisions among them and the interactions with the boundaries. The motions and the intermolecular collisions are uncoupled and the former are simulated deterministically and the latter are followed statistically. The method is expounded thoroughly in [2] by

* Present working address: Dept of Aerospace Engineering, Univ. of Michigan, Ann Arbor, MI 48109

CP585, Rarefied Gas Dynamics: 22 $2^{\text {nd }}$ International Symposium, edited by T. J. Bartel and M. A. Gallis

(C) 2001 American Institute of Physics 0-7354-0025-3/01/\$18.00 
Bird. In our simulation, the flow media is assumed to be a diatomic molecule gas with a molecule mass of $4.81 \times 10^{-26} \mathrm{~kg}$. The variable hard sphere or VHS model ${ }^{[2]}$ is employed to describe the collisions between molecules.

Singular circular microchannel of diameter $D$ (Fig.1) is used to imitate the performance of membrane filter. A ring diaphragm of inner diameter $d$ is fit in to give an appropriate opening factor $\beta=d^{2} / D^{2}$. The simulated sections of the channel upstream and downstream of the diaphragm AB and EF are chosen long enough to allow the flow to be established with a certain pressure drop. For small inner diameter $d$ the length $\mathrm{AB}$ or EF is of order $2.5 \sim 3 D$, for large $d$ the length is of order $1 D$. The walls of the diaphragm BCDE are assumed to be diffusely reflecting surface, while the side walls $\mathrm{AB}$ and $\mathrm{EF}$ are assumed to be specularly reflecting.

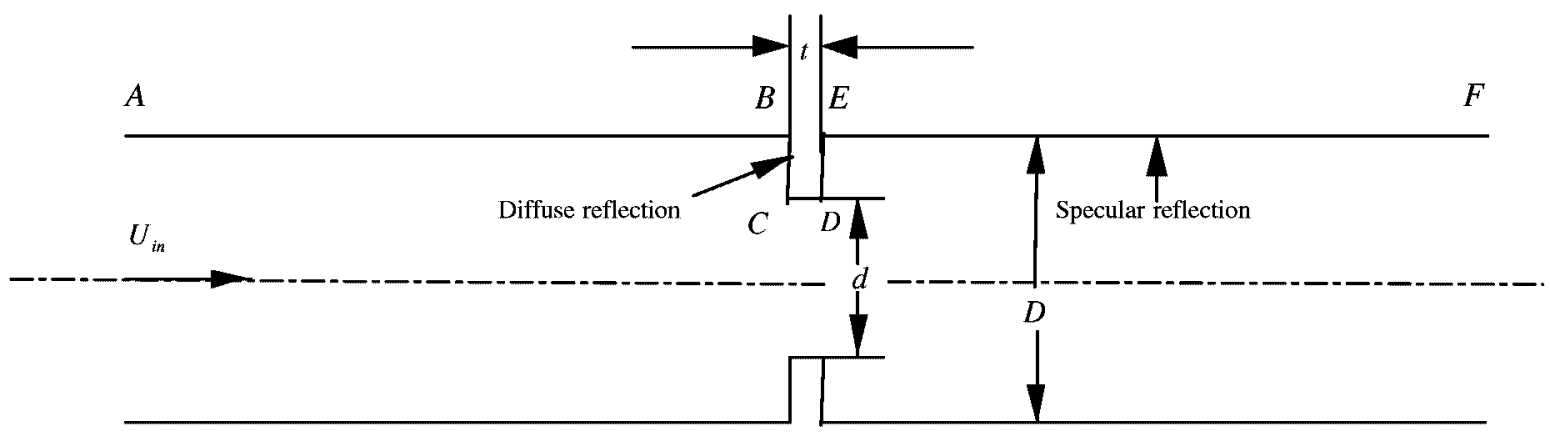

Fig. 1 The geometry of the microchannel

For boundary conditions of DSMC procedure in the subsonic flow case the particle number flux must be specified for each cell of the inlet and outlet cross sections of the microchannel. To specify the particle number flux, the number density (or pressure, under the isothermal conditions) and the macroscopic velocity are to be identified. But it is impossible to give both the pressure and velocity on both inlet and outlet of the channel flow reconcilably, unless the physically correct solution is obtained. The boundary values can be given only by trial and they are relaxed by the results obtained by DSMC simulation to give modified new boundary values. In the present problem the following boundary condition treatment is used. On the inlet and outlet of the channel the pressures are kept equal to constant values $p_{i n}$ and $p_{o u t}$, while the velocity distributions are adjusted gradually, being relaxed from the old distribution by the simulated result to a new distribution

$$
\mathbf{u}^{\text {new }}=\zeta \mathbf{u}^{\text {old }}+(1-\zeta) \mathbf{u}^{\text {simulated }}
$$

which approaches an established convergent result, where $\zeta$ is a relaxation factor, $0<\zeta<1$.

Gradually increasing the proportion of the magnitude of "old" molecular quantity along with the increase in sample size could help to implement the right choice of the value of the relaxation factor. The influence of the boundary condition modification could be introduced in different ways at different stage of flow field. In this paper, the same relaxation factor as in the boundary condition is adopted to calculate weighted average values of the whole flow field at each stage. The final results are obtained when convergence is reached.

As a global result of the DSMC calculation the dimensionless pressure drop factor 


$$
K=\frac{\Delta p}{\frac{1}{2} \rho U_{\text {in }}{ }^{2}} \beta^{2}(t / d)^{-0.28}
$$

is obtained as function of the Reynolds number based on the flow parameters at the hole

$$
\operatorname{Re}=\rho U_{h} d / \mu
$$

where $\Delta p=p_{\text {in }}-p_{\text {out }}, \rho$ is the density, $t$ is the thickness of the diaphragm, $U_{\text {in }}$ is the inlet velocity, $U_{h}$ is the average velocity of the gas through the hole. In Fig. 2 the DSMC simulation results of $K$ for circular section (?) with an inner diameter of the diaphragm $d=81 \mathrm{I} \mathrm{m}(\beta=8 \%)$ are given together with the experimental data ( $1, ?, ?)$ and the N-S (with slip condition) results (solid lines) of [1]. One can see that the DSMC simulation results are in excellent agreement with the experimental data for circular section with the same opening factor $(\beta=8 \%)$.

To study the effect of the shape of the channel and diaphragm on the performance of the filter, the flow through channel with rectangular section and diaphragm is simulated by a 3-D DSMC code. The dimensions of the rectangle section and diaphragm are $D_{1} \times D_{2}$ and $d_{1} \times d_{2}$ with $D_{1}: D_{2}=d_{1}: d_{2} . \beta$ for rectangular section $\left(d_{1} d_{2} / D_{1} D_{2}\right)$ is taken in the range from $12 \%$ to $70 \%$. The final results of flow field at each operating condition for rectangular section should be compared with those for circular section with the same $\beta$. The results of pressure drop ( $囚$ for $\beta=9 \%$ ) are given for square shape $\left(d_{1}=d_{2}\right)$ in Fig. 2. The pressure drop factor of the form (2) with an equivalent diameter $d=(4 / \pi)^{1 / 2} d_{1}$ is used.

One can see that the results of DSMC calculation for both circular and rectangular channels with appropriately defined opening factors of $8 \%$ and $9 \%$ are in general agreement with the experimental data for circular section

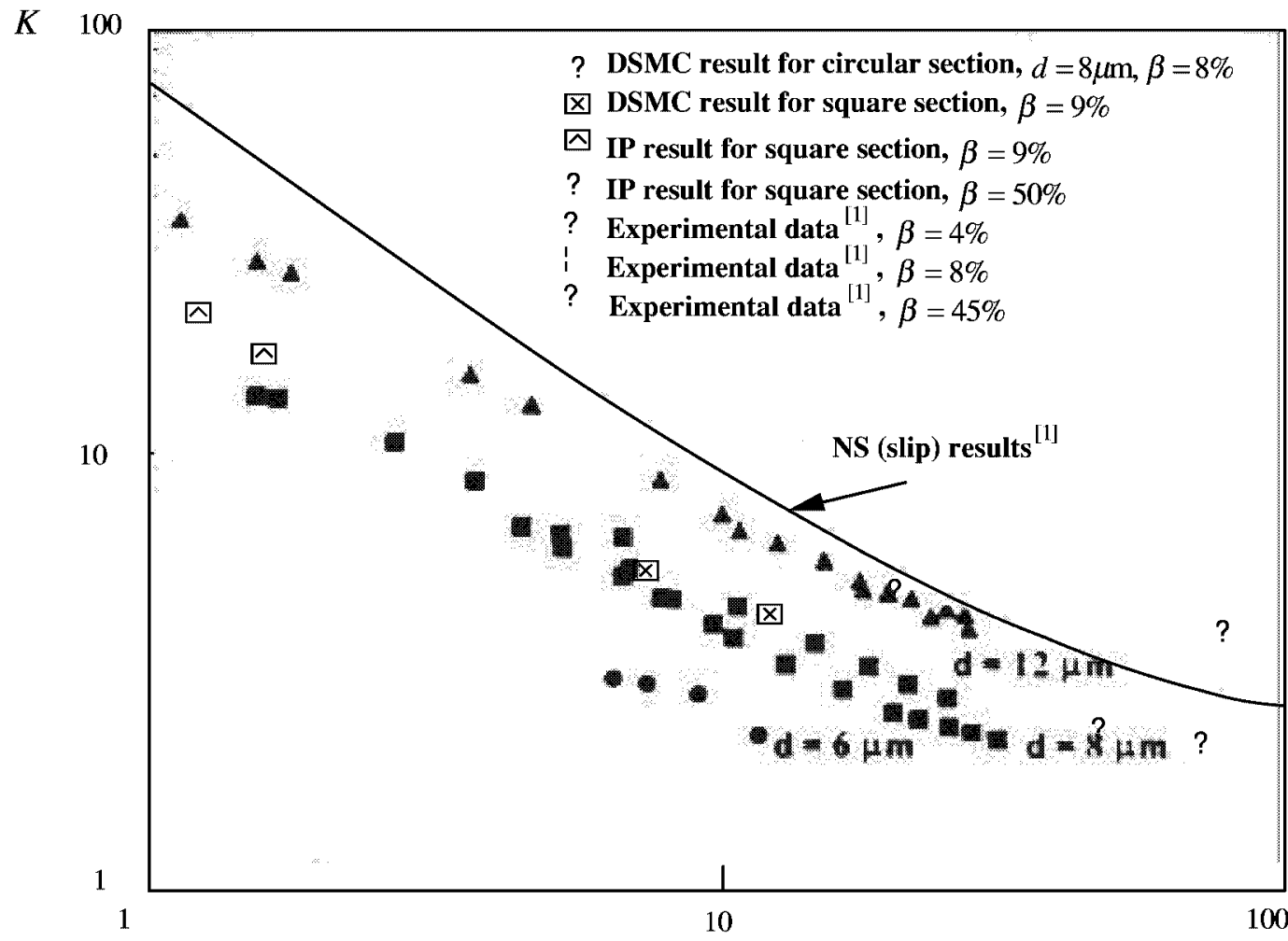

$\operatorname{Re}$

Fig. 2 The pressure drop factor obtained by the present paper in comparison with experimental data and the NS (slip) results 
with $\beta=8 \%$ of membrane filters with regularly arranged perforations. (The experimental data marked as i obtained in [1] include the results for hexagonal hole sections with $\beta=20 \%$, but we can not distinguish them from the data needed.)

It is worthwhile to note that the Knudsen number $(\lambda / d)$ of the filters studied in [1] and in our DSMC simulation ranges from 0.005 to 0.015 . It is the ideal realm for N-S equation with slip condition used in [1] to work. The deviation of numerical result of [1] from the experimental data and our DSMC calculation remains a perplexing problem of continuum fluid mechanics. In our DSMC simulation the sections AB and EF of wall have only the role to confine the flow, but specular reflection of molecules on them does not retard the flow. This explains the success of our DSMC simulation in this realm of $K n$.

In Fig. 3 the DSMC simulation results of flow field for both circular (a) and rectangular (b) sections are given.

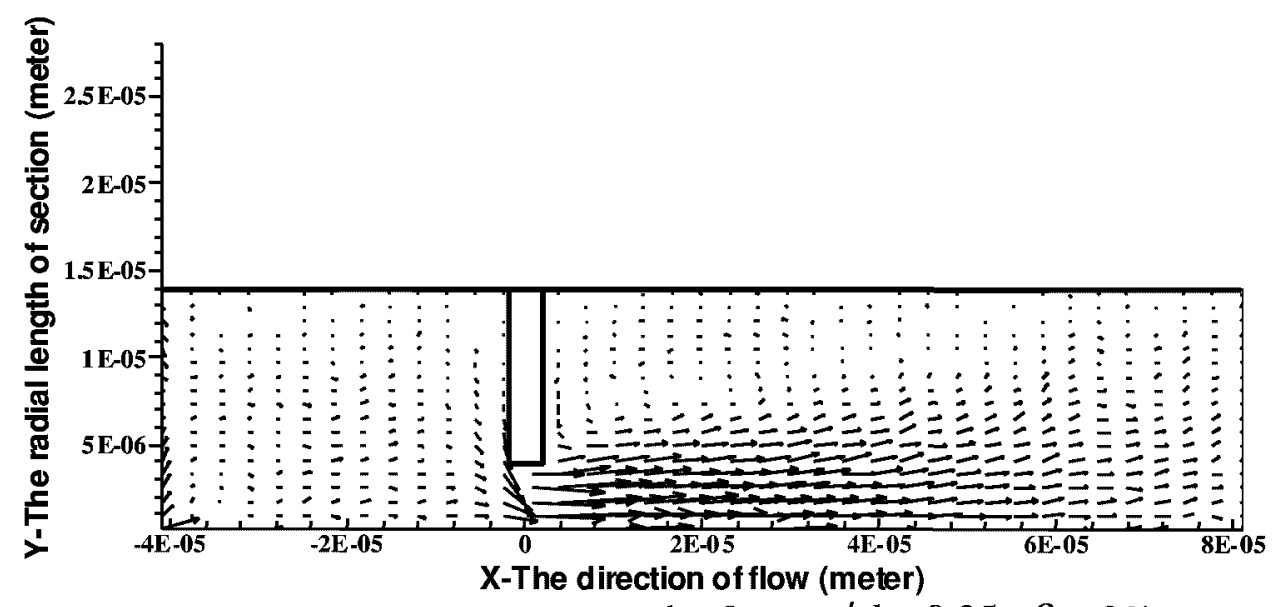

(a) Channel with circular section, $d=8 \mu \mathrm{m}, t / d=0.25, \beta=8 \%$

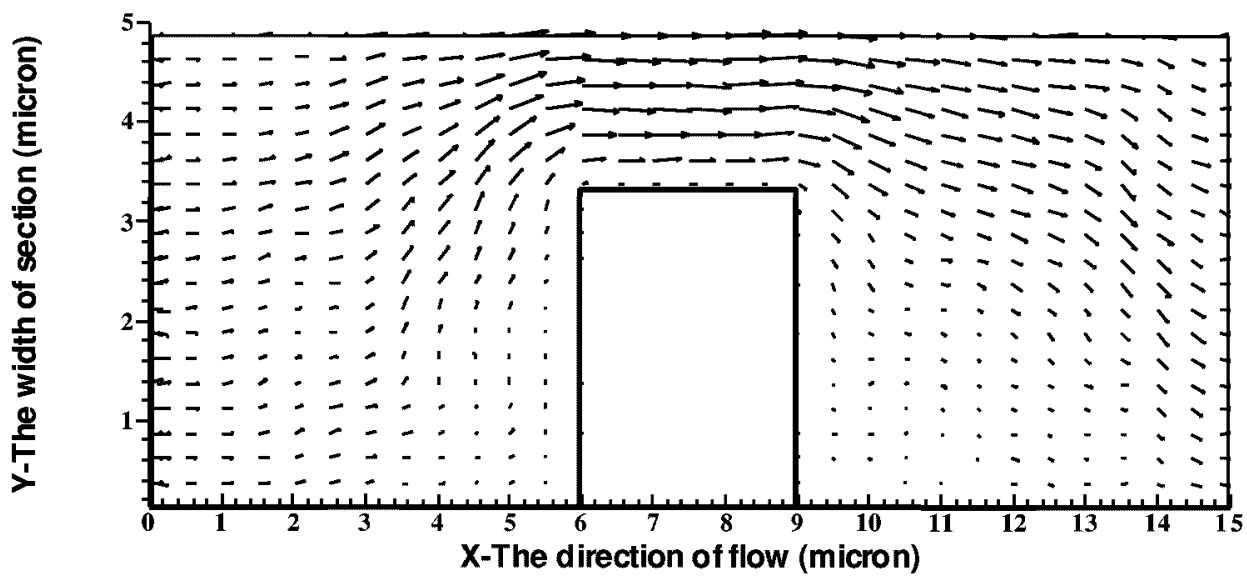

(b) Channel with square section, $t / d=1, \beta=9 \%$

Fig. 3 DSMC simulation results of flow field for both circular (a) and square (b)

sections, $n_{\text {inlet }}=2.69 \times 10^{25}, n_{\text {outlet }}=2.60 \times 10^{25}$ 


\section{INFORMATION PRESERVATION (IP) METHOD}

To overcome the difficulty of large noise to signal ratio in using the DSMC procedure to simulate the low speed flow in transitional regime, characteristic for the MEMS, the information preservation (IP) method is put forward in [3], which suggests to preserve some macroscopic information for a simulated molecule in DSMC simulation to carry, which reflects the collective behavior of the enormous number of real molecules it represents. In [4] the IP method was used to deal with the two-dimensional low speed rarefied gas flow around airfoils. Here on the example of channel flow with diaphragm the procedure to implement the IP method for three-dimensional case is demonstrated.

Except the usual microscopic velocity $\mathbf{c}$ used in DSMC method the IP velocity $\mathbf{u}$, the IP cell velocity $\mathbf{U}$ and cell density $\rho$ is introduced. $\rho$ and $\mathbf{U}$ are governed by the continuum and momentum equations

$$
\begin{aligned}
& \iiint \frac{\partial \rho}{\partial t} d V=-\iint \rho \mathbf{U} \cdot \mathbf{n} d A \\
& \iiint \rho \frac{D \mathbf{U}}{D t} d V=-\iint p \mathbf{n} d A
\end{aligned}
$$

From (4) and (5) one obtains the density and velocity increments of each cell

$$
\begin{aligned}
& \Delta \rho=-\frac{\Delta t}{\Delta V} \iint \rho \mathbf{U} \cdot \mathbf{n} d A \\
& \Delta \mathbf{U}=-\frac{\Delta t}{\rho \Delta V} \iint p \mathbf{n} d A
\end{aligned}
$$

The integrals are taken on the boundaries of that cell, and are calculated approximately as the sums of products of the under-integral value and the areas of the cell boundary. The increments of density and velocity are added to the corresponding IP values of simulated molecules, and the revised values are used to calculate the increments on the next time step. During collision of two simulated molecules a common post-collision IP velocity, the mass weighted average of the pre-collision velocities, is assigned to each of them:

$$
\mathbf{u}_{1}^{\prime}=\mathbf{u}_{2}^{\prime}=\frac{m_{1} \mathbf{u}_{1}+m_{2} \mathbf{u}_{2}}{m_{1}+m_{2}}
$$

where superscript ' denotes post-collision values. The essence of equation (8) is not in the inelastic collision rule but is in the conservation of momentum of large number of real molecules.

When reflecting from walls, IP velocity change the sign of its normal to the wall component leaving the rest components unchanged for specular reflection and gains the wall velocity for diffuse reflection. The calculation proceeds until convergence is gained.

The results of IP simulation for the case of square section channel ( $₫$ for $\beta=9 \%$ and ? for $\beta=50 \%$ ) are also shown in Fig. 2, and are seen to be in excellent agreement with the experimental data for $\beta=8 \%$ (1) and for $\beta=45 \%$ (?). The results of DSMC simulation for $\beta=8 \%$ (?, circular section) and for $\beta=9 \%$ (区, square section), and the IP results for $\beta=9 \%$ ( $囚$, square section) together with the experimental data (i) for $\beta=8 \%$ have the implication that the pressure drop factor can be characterized by a functional relationship of $\operatorname{Re}$ and $\beta$. The agreement of IP results with experimental data and also with DSMC simulation strengthens the confidence in using IP procedure to deal with 3-D low-speed flows encountered in MEMS. 
In order to economize the memory of computer and to increase calculating speed, in a DSMC simulation the initial status of microchannel flow field is assumed vacuum. Under any initial situation of two ends of the channel, the boundary conditions would always converge to values accommodated to the pressure difference and geometry of the diaphragmas long as the values of pressure upon both inlet and outlet of the microchannel are set. But it is not a suitable way to start the IP simulation from a vacuum state in the channel. A number of unrealistic situations would occur when start the IP process from certain conditions of the inlet and outlet of the channel. It is natural to start the IP simulation from a uniform flow field. But in this paper, as the main concern is to have the correct simulation results of DSMC and IP procedure but not to attain the maximum efficiency of the method, we just start the IP procedure from the well converged DSMC flow.

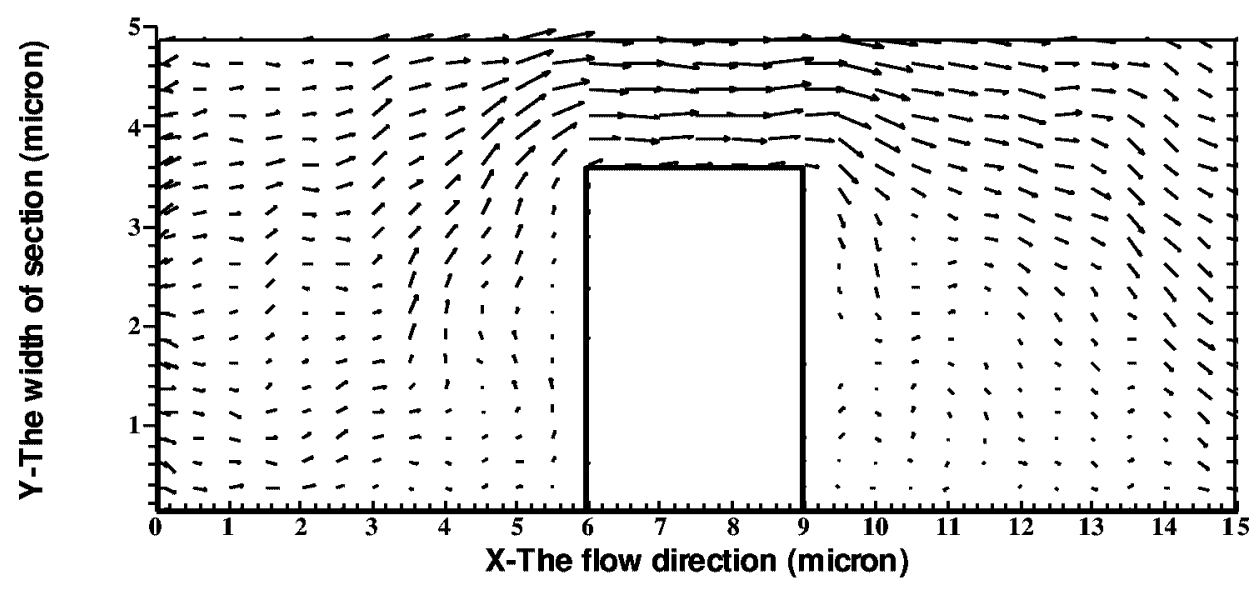

(a) DSMC result

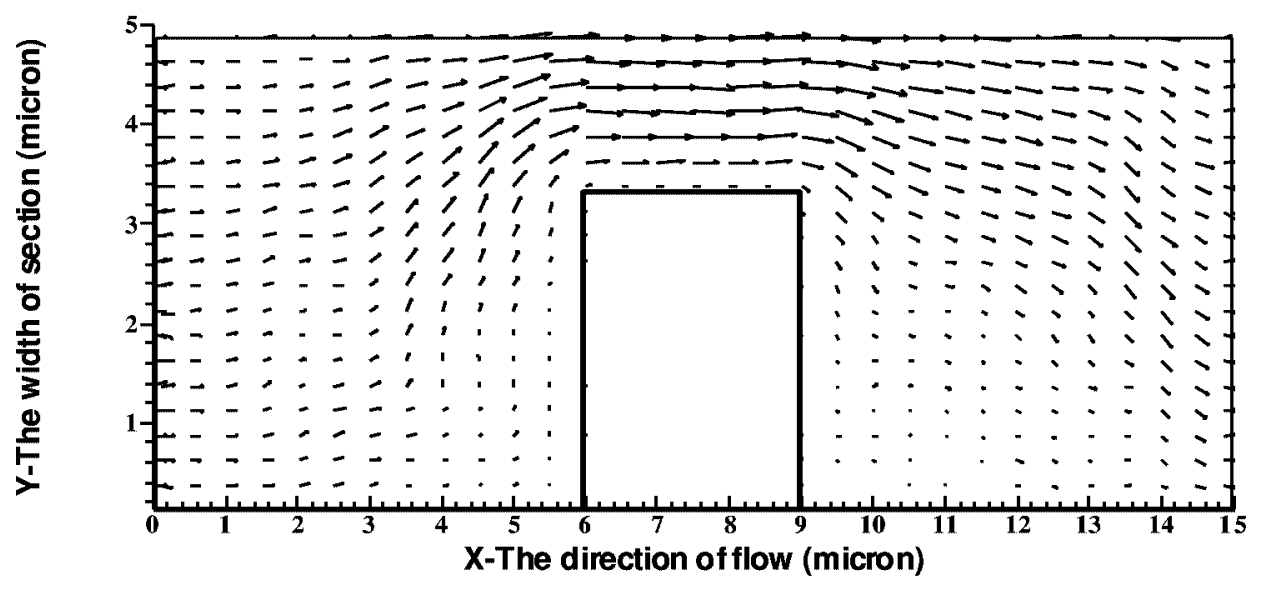

(b) IP result with DSMC sample as initial condition

Fig. 4 Comparison of DSMC (a) and IP (b) simulation, $t / d=1, \beta=9 \%, n_{\text {inlet }}=2.69 \times 10^{25}$,

$$
n_{\text {outlet }}=2.65 \times 10^{25}
$$


Then, fast convergent satisfactory results are obtained. This affirms the feasibility of using IP procedure to deal with 3-D low-speed flows involved in MEMS. Simulation shows that small pressure difference on the inlet and outlet of the channel could result in a large flow velocity, for example, $n_{\text {inlet }}=2.69 \times 10^{25}$ and $n_{\text {outlet }}=2.65 \times 10^{25}$ ( $n$ is the number density of the gas) corresponding to the average flow velocity $5 \mathrm{~m} / \mathrm{s}$. Therefore, higher precision and faster convergent speed of gas density are necessary.

The DSMC and IP simulation experience also shows that some small details of the flow characteristics might be ignored in IP with the main global feature remained. For example, Fig. 4 is the flow obtained by DSMC and IP methods for the same inlet and outlet conditions (very low-pressure difference). It shows that in the results of IP simulation the weak vortex appeared behind the diaphragm in the DSMC calculation when $\beta$ is relatively small could not be detected. The reasons of those phenomena may be that under low-pressure difference, the macroscopic summation of IP physical quantities would ignore some very small information. But the main results of some global flow quantity for low-gas velocity calculated by IP and DSMC appear to have very satisfactory agreement. So IP could be used to estimate the global flow quantity when the details of flow configuration is not of high importance.

\section{CONCLUSIONS}

The calculations of flow through micromachined membrane particle filter by statistic simulation on a model single microchannel flow with diaphragm and specularly reflecting side walls show excellent agreement with the experimental data. This shows the feasibility of the statistical simulation approach to solve the problem of performances of membrane particle filter.

It is still a challenge to clarify why discrepancy occurs between the experimental data and the numerical calculations of N-S equation with slip boundary condition for a Knudsen number $\lambda / d$ range of 0.005 to 0.015 , which is a ideal realm for $\mathrm{N}-\mathrm{S}$ equation with slip condition to work. Additional work in the continuum fluid dynamics aspects is to be done to resolve this knotty problem.

Preliminary information preservation (IP) simulations for 3-D membrane filter flow yield results in excellent agreement with the DSMC simulation results and experimental data. Differences in details of flow patterns obtained by DSMC and IP methods have been detected for some flow situations with vortices, but the global performance of the filter flow is not affected. The IP procedure deserves future development in enhancement of efficiency and simulation technique.

\section{ACKNOWLEDGMENT}

The support by NNSFC (grants $19772059,19889209,59876043$ ) is cordially appreciated by the authors.

\section{REFERENCES}

1. Yang, X., et al., Micromachined membrane particle filters, Proceeding of the $11^{\text {th }}$ Annual International Workshop on MicroElectro-Mechanical-Systems, Jan. $2^{\text {th }}-29^{\text {th }}$, Heidelberg, Germany, 1998, pp. 137-142.

2. Bird, G. A., Molecular Gas Dynamics and the Direct Simulation of Gas Flows, Clarendon Press, Oxford(1994).

3. Fan, J., and Shen, C., Statistical simulation of low speed unidirectional flows in transition regime, in Rarefied Gas Dynamics, ed. by R. Brun, R. Campargue, R. Gatignol, and J. C. Lengrand, vol. 2, 1999, pp. 245-252.

4. Fan, J., Boyd, I. D., and Cai, C. P., Computation of rarefied flows around a NACA 0012 airfoil, AIAA Paper 99-3804(1999). 